\title{
Elastic and viscoelastic properties of sugarcane bagasse-filled poly(vinyl chloride) composites
}

\begin{abstract}
Elastic and viscoelastic properties of sugarcane bagasse-filled poly(vinyl chloride) were determined by means of three-point bending flexural tests and dynamic mechanical and thermal analysis. The elastic modulus, storage modulus, loss modulus, and damping parameter of the composites at fibre contents of 10,20,30, and $40 \%$ in mass were determined, as well as those of the unfilled matrix. There was a correlation between the elastic modulus and storage modulus of the composites. Moreover, the elastic and viscoelastic properties of the composites were highly influenced by fibre content.
\end{abstract}

Keyword: Bagasse; Composite; Elasticity; Viscoelasticity; Thermomechanical 